\title{
A Relationship Between Unexplained Dizziness and Beta-amyloid Plaques Among Older Adults - Mayo Clinic Longitudinal Study of Aging
}

RAZAN AL FAKIR ( $\sim$ ralfakir69@gmail.com )

Auburn University https://orcid.org/0000-0002-9781-8006

Method Article

Keywords: Aging, Beta-amyloid plaque, Alzheimer's disease, Vestibular Functions

Posted Date: April 1st, 2021

DOl: https://doi.org/10.21203/rs.3.rs-384231/v1

License: (c) (i) This work is licensed under a Creative Commons Attribution 4.0 International License.

Read Full License 


\section{Abstract}

\section{Background and Objective}

Beta-amyloid $(A \beta)$ plaque deposition, which can be detected by amyloid $\mathrm{C}-11$ labeled Pittsburgh Compound $\mathrm{B}\left({ }^{11} \mathrm{C}-\mathrm{PiB}\right)$ Positron Emission Tomography (PET) imaging, is a key feature of Alzheimer's disease (AD) and occurs years before the onset of symptoms. It is well-documented that the onset of $A D$ initially involves degeneration of cholinergic systems in the posterior parietal-temporal, medial-temporal, and posterior-cingulate regions, which have a strong relationship with the vestibular labyrinth. In the context of an aging population, the symptoms-related vestibular system ranks among the most frequent complaints in primary care but remains unexplained in $40-80 \%$ of cases over the age of 65 . The objective of this study was to evaluate the cross-sectional and longitudinal association between the symptoms-related vestibular system and A $\beta$-PET imaging among older adults.

\section{Methods}

A retrospective study design. The study included 5707 participants without dementia, enrolled in the Mayo Clinic Study of Aging (mean age (SD) 74.4 (9.7) years) with cognitive evaluation and information about symptoms-related vestibular system, mainly dizziness or lightheadedness, at baseline and for every 15 months.

\section{Results}

Of the 5707, 924 (16\%) participants were complaining of 'unexplained' dizziness or lightheadedness. The estimated risk of developing symptoms of dizziness or lightheadedness at 10 years was $49 \%$. Data suggested that AD-related risk factors including, age, male gender, lower education, high comorbidity index, high-density lipoprotein, balance problems, presence of neurocognitive and neurobehavioral outcomes, and brain-related changes can contribute to the risk of developing 'unexplained' dizziness or lightheadedness among older adults. Adjusting for the risk factors, a statistically significant association between 'unexplained' dizziness or lightheadedness and A $\mathrm{P}$-PET imaging was evident $[H R=1.8, P=$ 0.009]. This association seems to be significantly modulated by psychological outcomes including, depressive symptomatology and anxiety $[\mathrm{HR}=1.0, \mathrm{P}=0.001]$.

\section{Conclusions}

'Unexplained' dizziness or lightheadedness was associated with $A \beta$ plaque deposition among older adults. This association seems to be modulated by psychological outcomes. Although these symptoms can be linked by a common cause, it may suggest functional dizziness, also known as psychogenic dizziness or persistent postural-perceptual dizziness. Further studies are needed to support the findings.

\section{Introduction}


Ageing is one of the most well-established risk factors for many organic or systemic diseases. In the context of aging population, it can be complicated enough to care for elderly patient with a single disease; however, this is not the case if we are aiming to prevent or delay the onset of with Alzheimer's disease (AD) or a related dementia (Alkadhi \& Eriksen, 2011; Masters \& Beyreuther, 1998). AD has emerged as the predominant form of dementia in the elderly, affecting one person in ten over the age of 65, and 50 percent of individuals over the age of 85 (Evans et al., 1989; Qiu, Kivipelto, \& von Strauss, 2009).

It is well-established that multiple chronic conditions, physical inactivity, and mental illness such as depression and anxiety can increase the risk of Beta-amyloid $(A \beta)$ plaques build-up (also known as neuritic plaques or senile plaques) in the areas of the brain concerned with memory in cognitively normal adults (Livingston et al., 2020; Rodrigues et al., 2012; Santos et al., 2017). If left unrecognized, A $\beta$ plaques deposition found to be associated with increased risk of decline on cognitive measures of visuospatial function, episodic and semantic memory, mental status, and increased risk of progression to $A D$ (Livingston et al., 2020; Mormino \& Papp, 2018; Murphy \& LeVine, 2010). Hence, AD pathogenesis is widely believed to be driven by the presence of $A \beta$ plaques in the aggregated brain regions (Murphy \& LeVine, 2010). These regions involve degeneration of cholinergic systems in the posterior parietaltemporal, medial-temporal, and posterior-cingulate regions, which have a strong relationship with the vestibular labyrinth (Previc, 2013).

Neuroimaging studies have found that patients with preclinical AD have widespread white matter abnormalities at a stage like those reported in Alzheimer's disease, whereas gray matter structures were relatively intact. In addition, widespread white matter abnormalities o is reported to occur prior to the presence of amyloid- $\beta$ plaques (Sachdev, Zhuang, Braidy, \& Wen, 2013). Remarkably, C-11 labeled Pittsburgh Compound B $\left({ }^{11} \mathrm{C}-\mathrm{PiB}\right)$ was the first successful Positron Emission Tomography (PET) is the leading neuroimaging tool to detect and provide quantitative measures of $A \beta$ plaques in vivo at the early stages and follow its course longitudinally (Villemagne, Doré, Burnham, \& Rowe, 2021; Vlassenko, Benzinger, \& Morris, 2012; Wiley et al., 2009).

Dizziness is frequently described as a symptom of peripheral vestibular loss or a consequence or side effect of defined entities such as cardiovascular, infectious, and neurological disease or as a discrete syndrome associated with co-morbidities (Neuhauser et al., 2008). Dizziness is a subjective perception of disorientation or involuntary motion that occurs during head or body movement or when the head or body is still. Dizziness can be further characterized as lightheadedness, which is the sensation of impending loss of consciousness associated with transient diffuse cerebral hypoperfusion. The severity of dizziness or lightheadedness is a potentially disabling condition that has a distinct impact on physical activity, mental health, and cognitive status since it is provoked by postural change and head and neck movement (Sloane, Hartman, \& Mitchell, 1994; Tinetti, Williams, \& Gill, 2000).

Dizziness ranks among the most frequent complaints in primary care but remains unexplained in $40-$ $80 \%$ of cases over the age of 65 and typically lacks uniform criteria for its classification and definition, 
especially when not better accounted for by vestibular diagnosis (Bösner et al., 2018; Iwasaki \& Yamasoba, 2014). A study found that cerebral atrophy was found in $86 \%$ of dizzy subjects and $85 \%$ controls and at least one white matter lesion was found in $69 \%$ of dizzy subjects and $78 \%$ controls; however, white matter lesions in the midbrain were significantly common in dizzy than in non-dizzy subjects (Colledge et al., 2002). This raises a concern of a potential link between 'unexplained' dizziness or lightheadedness and presence of $A \beta$ plaques. The objective of this study was to evaluate the crosssectional and longitudinal association between symptoms-related vestibular system and A $\beta$-PET imaging among older adults.

\section{Methods}

\section{Study design}

A retrospective study design was performed at the Mayo Clinic Florida. The Mayo Clinic Longitudinal Study of Aging Data Set (MCSA; (Roberts et al., 2008) was used to evaluate the cross-sectional and longitudinal association between dizziness or lightheadedness and Aß-PET imaging. The MCSA was approved by the Institutional Review Boards and the Olmsted Medical Center. The study design and methodology of the MCSA have previously been published in detail. Briefly, eligible participants of the MCSA were non-demented and $\geq 50$ years of age, with a concurrent, valid neurocognitive testing, neurobehavioral evaluation, and 11C-PiB PET imaging. The objective of the MCSA study was to establish a prospective population-based cohort to investigate (1) the prevalence of mild cognitive impairment; (2) the incidence of mild cognitive impairment; (3) conversion rates from mild cognitive impairment to dementia or $A D$; (4) risk factors for mild cognitive impairment; and (5) risk factors for the progression from mild cognitive impairment to dementia or $A D$.

\section{Participants}

Among 5707 participants in the MCSA, 924 participants who reported a mild to severe degree of dizziness or lightheadedness were selected. There were 483 (52.3\%) male and 441 (47.7\%) female participants with a mean age of 74.4 (9.7). The medical records for dizzy patients showed a non-organic origin of dizziness or lightheadedness. Demographics were compared between those with/without dizziness or lightheadedness using Wilcoxon signed-rank test or $\chi 2$ test as appropriate. Full details are presented in Table 1.

Out of those 5707 , the total follow-up time was 14.5 years. For simplification, the 14 years were divided into five events ( 1 year $+/$ - 6months; 3 years; 5 years; 7 years; and $10+/-2$ years). Wilcoxon signed-rank test was used to determine if there was a significant difference in score between baseline and the followup measurements. The $\chi 2$ test was used to compare categorical variables. Table 2 showed the mean difference between the baseline scores of neurocognitive evaluations and each event. P-value of $<0.05$ is considered significant. 
Table 1

Participants' characteristics by significant dizziness or lightheadedness at baseline

\begin{tabular}{|c|c|c|c|c|}
\hline & \multirow{2}{*}{$\begin{array}{l}\text { No }(\mathrm{N}=4758)^{a} \\
\text { Mean (SD); } \\
\text { Median }\end{array}$} & \multirow{2}{*}{$\begin{array}{l}\text { Yes (N= 924) } \\
\text { Mean (SD); } \\
\text { Median }\end{array}$} & \multirow{2}{*}{$\begin{array}{l}\text { Total }(N=5707) \\
\text { Mean (SD); } \\
\text { Median }\end{array}$} & \multirow{2}{*}{$\underset{b}{P \text {-value }}$} \\
\hline & & & & \\
\hline Age, at baseline & $72.9(10.1) ; 73.9$ & $74.4(9.7) ; 75.7$ & $73.2(10.0) ; 74.2$ & 0.001 \\
\hline Sex, male & $2406(50.6 \%)$ & $483(52.3 \%)$ & $2895(50 \%)$ & 0.343 \\
\hline Education, years & $14.4(2.8) ; 12.0$ & $14.0(2.8) ; 14.0$ & $14.3(2.8) ; 14.0$ & 0.001 \\
\hline Race, white & $4635(98.2 \%)$ & $896(97.7 \%)$ & $5096(89 \%)$ & 0.366 \\
\hline Ethnicity, Not Hispanic & $4686(99.7 \%)$ & $903(99.3 \%)$ & $5156(90 \%)$ & 0.094 \\
\hline $\begin{array}{l}\text { Charlson comorbidity } \\
\text { index }\end{array}$ & $3.0(3.0) ; 2.0$ & $3.7(3.3) ; 3.0$ & $3.1(3.1) ; 2.0$ & 0.001 \\
\hline APOE $\varepsilon 4$ positive & $1181(27.1 \%)$ & $256(29.5 \%)$ & $1441(25 \%)$ & 0.146 \\
\hline Lipid test (HDL) & $45.7(13.9)$ & $45.1(14.9)$ & $39(22)$ & 0.233 \\
\hline Balance difficulties & $721(15.5 \%)$ & $249(27.4 \%)$ & $764(13 \%)$ & 0.001 \\
\hline Hearing Loss at baseline & $1494(31 \%)$ & 367 (39\%) & $1868(32 \%)$ & 0.001 \\
\hline Gait speed $\leq 0.6 \mathrm{~m} / \mathrm{sec}^{b}$ & $144(30 \%)$ & $42(4.5)$ & $187(3.2)$ & 0.012 \\
\hline Beck Depression Inventory & $4.4(4.5) ; 3.0$ & $8.1(6.4) ; 7.0$ & $4.9(5.0) ; 4.0$ & 0.001 \\
\hline Beck Anxiety Inventory & $2.0(2.9) ; 1.0$ & $8.0(6.5) ; 6.0$ & $2.9(4.3) ; 1.0$ & 0.001 \\
\hline Global cognitive z-score ${ }^{c}$ & $-0.2(1.2) ;-0.2$ & $-0.6(1.2) ;-0.6$ & $-0.4(1.2) ;-0.3$ & 0.001 \\
\hline Memory z-score ${ }^{c}$ & $-0.4(1.2) ;-0.3$ & $-0.6(1.2) ;-0.6$ & $-0.4(1.2) ;-0.4$ & 0.001 \\
\hline Language $z$-score $^{c}$ & $-0.3(1.2) ;-0.2$ & $-0.5(1.3) ;-0.4$ & $-0.3(1.2) ;-0.2$ & 0.001 \\
\hline $\begin{array}{l}\text { Attention/executive z- } \\
\text { score }^{c}\end{array}$ & $-0.3(1.2) ;-0.1$ & $-0.7(1.4) ;-0.5$ & $-0.3(1.1) ;-0.2$ & 0.001 \\
\hline $\begin{array}{l}\text { Visuospatial skills z-score } \\
\mathrm{c}\end{array}$ & $-0.2(1.1) ;-0.1$ & $-0.4(1.1) ;-0.3$ & $-0.2(1.1) ;-0.3$ & 0.001 \\
\hline$A \beta-P E T$ imaging & $1.5(0.3) ; 1.4$ & $1.5(0.3) ; 1.4$ & $1.4(0.2) ; 1.4$ & 0.159 \\
\hline $\begin{array}{l}\text { AD signature thickness, } \\
\mathrm{mm}\end{array}$ & $2.7(0.2) ; 2.7$ & $2.7(0.2) ; 2.7$ & $2.6(0.1) ; 2.7$ & 0.077 \\
\hline
\end{tabular}

Note: data presented as $\mathrm{N}(\%)$ for categorical and mean (SD); median for continuous characteristics. ${ }^{\text {a }} 25$ missing. ${ }^{b} 465$ missing data. ${ }^{d}$ Global cognitive z-score was computed after scaling raw cognitive test 
scores (mean $0 \pm 1$ ) using data for cognitively unimpaired participants at baseline. Domain-specific zscores were summed and scaled to obtain global z-scores. 
Table 2

Neurocognitive evaluation by follow-up events as compared to baseline.

\begin{tabular}{|c|c|c|c|c|c|c|}
\hline Variables at baseline & $\begin{array}{l}\text { Baseline } \\
\text { Mean } \\
\text { (SD); } \\
\text { Median }\end{array}$ & $\begin{array}{l}1 \text { year } \\
\text { Mean } \\
\text { (SD); } \\
\text { Median }\end{array}$ & $\begin{array}{l}3 \text { years } \\
\text { Mean } \\
\text { (SD); } \\
\text { Median }\end{array}$ & $\begin{array}{l}5 \text { years } \\
\text { Mean } \\
\text { (SD); } \\
\text { Median }\end{array}$ & $\begin{array}{l}7 \text { years } \\
\text { Mean } \\
\text { (SD); } \\
\text { Median }\end{array}$ & $\begin{array}{l}10 \text { years } \\
\text { Mean } \\
\text { (SD); } \\
\text { Median }\end{array}$ \\
\hline \multirow[t]{3}{*}{$\begin{array}{l}\text { Beck Depression } \\
\text { Inventory }\end{array}$} & $\begin{array}{l}(\mathrm{N}= \\
5590)\end{array}$ & $\begin{array}{l}(\mathrm{N}= \\
3919)\end{array}$ & $\begin{array}{l}(N= \\
3569)\end{array}$ & $\begin{array}{l}(\mathrm{N}= \\
2702)\end{array}$ & $\begin{array}{l}(N= \\
1731)\end{array}$ & $(\mathrm{N}=783)$ \\
\hline & $5.0(5.1)$ & $\underbrace{4.7}_{\star \star}(4.9)$ & $4.6(4.7)$ & ${ }_{\star \star}^{4.8}(4.7)$ & $5.0(4.9)$ & $\underset{\star \star}{5.6}(5.2)$ \\
\hline & 4.0 & 3.0 & 3.0 & 4.0 & 4.0 & 4.0 \\
\hline \multirow[t]{3}{*}{ Beck Anxiety Inventory } & $\begin{array}{l}(\mathrm{N}= \\
5682)\end{array}$ & $\begin{array}{l}(\mathrm{N}= \\
3938)\end{array}$ & $\begin{array}{l}(\mathrm{N}= \\
3583)\end{array}$ & $\begin{array}{l}(\mathrm{N}= \\
2715)\end{array}$ & $\begin{array}{l}(N= \\
1753)\end{array}$ & \multirow{2}{*}{$3.0(4.7)$} \\
\hline & $2.9(4.4)$ & $\underset{\star *}{2.7}(4.0)$ & $\underset{\star \star}{2.7}(4.1)$ & $2.7(4.2)$ & $2.8(4.3)$ & \\
\hline & 1,0 & 1.0 & & 1.0 & 1.0 & 1.0 \\
\hline \multirow[t]{3}{*}{$\begin{array}{l}\text { Global cognitive z- } \\
\text { score } \mathrm{e}^{\text {a }}\end{array}$} & $\begin{array}{l}(\mathrm{N}= \\
5280)\end{array}$ & $\begin{array}{l}(\mathrm{N}= \\
3650)\end{array}$ & $\begin{array}{l}(\mathrm{N}= \\
3274)\end{array}$ & $\begin{array}{l}(\mathrm{N}= \\
2439)\end{array}$ & $\begin{array}{l}(N= \\
1559)\end{array}$ & \multirow{2}{*}{$\begin{array}{l}(\mathrm{N}=64) \\
-0.6(1.2) \\
\star \star\end{array}$} \\
\hline & $-0.4(1.2)$ & $\begin{array}{l}-0.2(1.3) \\
\star *\end{array}$ & $\begin{array}{l}-0.2(1.3) \\
\star \star\end{array}$ & $\begin{array}{l}-0.1(1.3) \\
\star \star\end{array}$ & $-0.2(1.2)$ & \\
\hline & -0.3 & -0.03 & 0 & 0.1 & 0 & -0.4 \\
\hline \multirow[t]{3}{*}{ Memory z-score ${ }^{\mathrm{e}}$} & $\begin{array}{l}(\mathrm{N}= \\
5609)\end{array}$ & $\begin{array}{l}(\mathrm{N}= \\
3884)\end{array}$ & $\begin{array}{l}(\mathrm{N}= \\
3530)\end{array}$ & $\begin{array}{l}(\mathrm{N}= \\
2651)\end{array}$ & $\begin{array}{l}(N= \\
1709)\end{array}$ & \multirow{3}{*}{$\begin{array}{l}(N=768) \\
-0.3(1.3) \\
-0.2\end{array}$} \\
\hline & $-0.4(1.2)$ & $\underset{\star *}{-0.1}(1.2)$ & $\begin{array}{l}-0.1 \\
\star \star\end{array}$ & $\underset{* \star}{0.0}(1.3)$ & $\underset{\star \star}{0.0}(1.3)$ & \\
\hline & -0.4 & -0.01 & 0.1 & 0.2 & 0.2 & \\
\hline \multirow[t]{3}{*}{ Language $z$-score ${ }^{\mathrm{e}}$} & $\begin{array}{l}(\mathrm{N}= \\
5488)\end{array}$ & $\begin{array}{l}(\mathrm{N}= \\
3801)\end{array}$ & $\begin{array}{l}(\mathrm{N}= \\
3432)\end{array}$ & $\begin{array}{l}(\mathrm{N}= \\
2586)\end{array}$ & $\begin{array}{l}(\mathrm{N}= \\
1674)\end{array}$ & $(N=752)$ \\
\hline & $-0.4(1.3)$ & $-0.2(1.2)$ & $-0.2(1.3)$ & $-0.2(1.3)$ & $-0.3(1.2)$ & $-0.7(1.3)$ \\
\hline & -0.2 & -0.1 & -0.01 & -0.1 & -0.1 & -0.4 \\
\hline \multirow[t]{3}{*}{$\begin{array}{l}\text { Attention/executive z- } \\
\text { score }{ }^{\mathrm{e}}\end{array}$} & $\begin{array}{l}(\mathrm{N}= \\
5437)\end{array}$ & $\begin{array}{l}(\mathrm{N}= \\
3745)\end{array}$ & $\begin{array}{l}(\mathrm{N}= \\
3379)\end{array}$ & $\begin{array}{l}(\mathrm{N}= \\
2522)\end{array}$ & $\begin{array}{l}(\mathrm{N}= \\
1620)\end{array}$ & $\begin{array}{l}(\mathrm{N}= \\
706) 0.9 \\
(1.2) * *\end{array}$ \\
\hline & $-0.4(1.3)$ & $-0.3(1.3)$ & $-0.3(1.3)$ & $\begin{array}{l}-0.3(1.3) \\
\star \star\end{array}$ & $-0.4(1.3)$ & \multirow{2}{*}{-0.6} \\
\hline & -0.2 & -0.01 & -0.1 & -0.1 & -0.3 & \\
\hline
\end{tabular}




\begin{tabular}{|c|c|c|c|c|c|c|}
\hline \multirow[t]{2}{*}{ Variables at baseline } & Baseline & 1 year & 3 years & 5 years & 7 years & 10 years \\
\hline & $\begin{array}{l}\text { Mean } \\
\text { (SD); } \\
\text { Median }\end{array}$ & $\begin{array}{l}\text { Mean } \\
\text { (SD); } \\
\text { Median }\end{array}$ & $\begin{array}{l}\text { Mean } \\
\text { (SD); } \\
\text { Median }\end{array}$ & $\begin{array}{l}\text { Mean } \\
\text { (SD); } \\
\text { Median }\end{array}$ & $\begin{array}{l}\text { Mean } \\
\text { (SD); } \\
\text { Median }\end{array}$ & $\begin{array}{l}\text { Mean } \\
\text { (SD); } \\
\text { Median }\end{array}$ \\
\hline \multirow[t]{3}{*}{$\begin{array}{l}\text { Visuospatial skills z- } \\
\text { score }^{\text {e }}\end{array}$} & $\begin{array}{l}(\mathrm{N}= \\
5426)\end{array}$ & $\begin{array}{l}(N= \\
3752)\end{array}$ & $\begin{array}{l}(\mathrm{N}= \\
3362)\end{array}$ & $\begin{array}{l}(N= \\
2511)\end{array}$ & $\begin{array}{l}(\mathrm{N}= \\
1604)\end{array}$ & $(N=706)$ \\
\hline & $-0.3(1.1)$ & $-0.1(1.1)$ & $-0.1(1.1)$ & $0.0(1.1)$ & $-0.0(1.1)$ & $* *$ \\
\hline & -0.2 & 0 & -0.1 & 0.1 & 0.1 & -0.2 \\
\hline \multirow[t]{3}{*}{$A \beta-P E T$ imaging } & $(\mathrm{N}=866)$ & $(\mathrm{N}=459)$ & $(N=538)$ & $(\mathrm{N}=359)$ & $(N=140)$ & $(N=78)$ \\
\hline & $1.5(0.3)$ & $\underset{* \star}{1.5}(0.3)$ & ${ }_{\star \star \star} .6(0.4)$ & $1.7(0.4)$ & $1.8(0.5)$ & $1.9(0.5)^{c}$ \\
\hline & 1.4 & 1.4 & 1.4 & 1.5 & 1.7 & 1.7 \\
\hline \multirow[t]{3}{*}{$\begin{array}{l}\text { AD signature thickness, } \\
\mathrm{mm}\end{array}$} & $\begin{array}{l}(N= \\
1831)\end{array}$ & $\begin{array}{l}(N= \\
1152)\end{array}$ & $\begin{array}{l}(N= \\
1216)\end{array}$ & $(\mathrm{N}=511)$ & $(\mathrm{N}=511)$ & $(N=78)$ \\
\hline & $2.7(0.2)$ & $2.6(0.2)$ & $2.6(0.2)$ & $\underset{\star \star \star}{2.6}(0.2)$ & $\underset{\star \star}{2.6}(0.2)$ & $\underset{\star \star}{2.5}(0.2)$ \\
\hline & 2.7 & 2.6 & 2.6 & 2.6 & 2.6 & 2.6 \\
\hline
\end{tabular}

\section{Statistical analysis}

For the analytical assessment of neurocognitive testing and ${ }^{11} \mathrm{C}-\mathrm{PiB}$ PET imaging, the raw scores or tests in each cognitive domain were z-scored, averaged, and scaled to create domain-specific cognitive zscores. Besides, a global z-score for overall cognitive performance was also created by averaging and scaling the four-domain z-scores. Hazard ratios (HR) for potential risk factors for each of the follow-up endpoints were obtained using Cox proportional hazards models. Univariate as well as multivariate models were assessed. Multivariate relationships were evaluated adjusting for age, sex, years of education, and other comorbidities. All analyses were considered statistically significant at a P-value < 0.05 and were performed using the SAS statistical software version 9.4 (SAS Institute, Cary, North Carolina).

\section{Results}

The risk of dizziness or lightheadedness during the follow-up period after the initial visit to the study was estimated using the Kaplan-Meier method. The estimated risk of developing symptoms of dizziness or lightheadedness at 10 years was $49 \%$. Figure 1 represents the survival curve. Potential risk factors to severity of dizziness or lightheadedness over time are presented in Table 3. Adjusting for age, sex, education, comorbidity (Charlson comorbidity index), and other risk factors, a statistically significant association between dizziness or lightheadedness and $A \beta-P E T$ imaging was evident $[H R=1.8, P=0.009]$. 
This association seems to be significantly modulated by neurobehavioral outcomes including depressive symptomatology and anxiety [HR $=1.0, P=0.001]$. Results are presented in Table 4.

Table 3

Factors contributing to dizziness or lightheadedness in older adults.

\begin{tabular}{|c|c|c|c|c|}
\hline Variables & \# of patients & HR & $95 \% \mathrm{Cl}$ & $P$-value \\
\hline Age at visit & 4783 & 1.02 & $1.01-1.03$ & 0.001 \\
\hline Sex, male & 4783 & 1.13 & $1.01-1.28$ & 0.032 \\
\hline Education, years & 4778 & 0.95 & $0.93-0.97$ & 0.001 \\
\hline Race, white & 4745 & 0.89 & $0.57-1.41$ & 0.640 \\
\hline Ethnicity, Not Hispanic & 4723 & 1.11 & $0.35-3.45$ & 0.852 \\
\hline Charlson comorbidity index & 4782 & 1.07 & $1.05-1.09$ & 0.001 \\
\hline APOE $\varepsilon 4$ positive & 4381 & 1.095 & $0.95-1.25$ & 0.640 \\
\hline Lipid test (HDL) & 1543 & 0.99 & $0.98-0.99$ & 0.008 \\
\hline Balance difficulties & 4654 & 1.07 & $1.06-1.08$ & 0.001 \\
\hline Gait speed $\leq 0.6 \mathrm{~m} / \mathrm{sec}^{d}$ & 4410 & 1.30 & $0.93-1.83$ & 0.130 \\
\hline Beck Depression Scale (BDS) & 4684 & 1.07 & $1.06-1.08$ & 0.001 \\
\hline Beck Anxiety Inventory (BAI) & 4758 & 1.08 & $1.07-.10$ & 0.001 \\
\hline Global cognitive z-score ${ }^{\mathrm{e}}$ & 4426 & 0.83 & $0.79-0.88$ & 0.001 \\
\hline Memory z-score ${ }^{e}$ & 4702 & 0.85 & $0.80-0.89$ & 0.001 \\
\hline Language $z$-score ${ }^{\mathrm{e}}$ & 4600 & 0.86 & $0.82-0.91$ & 0.001 \\
\hline Attention/executive $z$-score ${ }^{\mathrm{e}}$ & 4553 & 0.84 & $0.79-0.88$ & 0.001 \\
\hline Visuospatial skills z-score ${ }^{\mathrm{e}}$ & 4552 & 0.89 & $0.85-0.95$ & 0.003 \\
\hline$A \beta-P E T$ imaging & 744 & 2.23 & $1.45-3.44$ & 0.002 \\
\hline AD signature thickness, $\mathrm{mm}$ & 1548 & 0.38 & $0.19-0.74$ & 0.004 \\
\hline
\end{tabular}


Table 4

Association between dizziness or lightheadedness and A $\beta$-PET imaging

\begin{tabular}{|c|c|c|c|c|}
\hline Outcomes & \# of patients & HR & $95 \% \mathrm{Cl}$ & $P$-value \\
\hline Beck Depression Scale & 4680 & 1.07 & $1.05-1.08$ & 0.001 \\
\hline Beck Anxiety Inventory & 4753 & 1.08 & $1.06-.10$ & 0.001 \\
\hline Global cognitive $z$-score ${ }^{e}$ & 4425 & 0.94 & $0.88-1.01$ & 0.108 \\
\hline Memory z-score ${ }^{\mathrm{e}}$ & 4701 & 0.94 & $0.88-1.01$ & 0.106 \\
\hline Language $z$-score ${ }^{\mathrm{e}}$ & 4599 & 0.95 & $0.91-1.01$ & 0.170 \\
\hline Attention/executive z-score ${ }^{\mathrm{e}}$ & 4552 & 0.95 & $0.89-1.01$ & 0.147 \\
\hline Visuospatial skills z-score ${ }^{\mathrm{e}}$ & 4551 & 1.00 & $0.93-1.06$ & 0.991 \\
\hline$A \beta-P E T$ imaging & 743 & 1.87 & $1.16-3.02$ & 0.009 \\
\hline$A D$ signature thickness, mm & 1547 & 1.20 & $0.54-2.68$ & 0.650 \\
\hline
\end{tabular}

\section{Discussion}

To the best of our knowledge, this is the first study to evaluate the relationship between dizziness or lightheadedness, that not better accounted for by peripheral vestibular diagnosis and A $-P E T$ imaging among older adults. The data highlighted the risk of developing dizziness or lightheadedness overtime. Also, data suggested that AD-related risk factors including, age, male gender, lower education, high comorbidity index, high-density lipoprotein, balance problems, presence of neurocognitive and neurobehavioral outcomes, and brain-related changes can contribute to the prevalence of dizziness or lightheadedness among older adults. In addition, after controlling for the risk factors including neurocognitive and neurobehavioral outcomes, our model revealed a significant relationship between dizziness or lightheadedness and A $\beta$-PET imaging among older adults, in which neurobehavioral outcomes (depressive symptomatology and anxiety) appear to be intimately related to this relationship. This relationship may emphasize the complex interactions of different pathways underlying both depression, dizziness or lightheadedness, and A $\beta$-PET imaging. Besides what was being highlighted in the introduction section, there are several reasons that intuitively support our findings.

First, anxiety and depression, on one hand, and dizziness or lightheadedness, on the other, are common complaints among elderly people. Several studies underlined that depressive symptomatology and anxiety can be symptoms of underlying health problems, such as dizziness or lightheadedness, and vice versa (Best, Eckhardt-Henn, Tschan, \& Dieterich, 2009; Carmeli, 2015; Kotova \& Zamergrad, 2016).

Second, a previous study using the Baltimore Longitudinal Study of Aging examined a relationship between peripheral vestibular loss, in which dizziness is a common symptom, and the $A \beta$ deposition 
(Kamil, Bilgel, Wong, Resnick, \& Agrawal, 2018). However, the study did not observe a significant relationship between measures of vestibular function and A $\beta$-PET imaging in cognitively intact older adults. Similarly, a study investigated whether current depressive symptoms are related to cortical $A \beta$ deposition (Chung et al., 2016). They found that current depressive symptoms were not related to cortical $A \beta$, after controlling for potential confounds, including the history of major depression. They also observed that there was no difference in cortical $A \beta$ between matched participants with high and low depressive symptoms, as well as no difference between matched participants with the presence and absence of depressive symptoms.

Third, the vestibular system was considered as a sixth sense as it contributes to a wide range of functions from the level of reflexes to the level of cognition and coordination. Connections between the vestibular system and cognitive functions have attracted much recent interest over the last century, motivated by emerging evidence that impaired vestibular function is a risk factor for cognitive decline. However, dementia and reduced cognitive function present immense challenges, and their intersection remain poorly understood and difficult to assess, even in the presence of $A \beta$ plagues (Kamil et al., 2018). Typically, elderly individuals complain of persistent dizziness or lightheadedness even before the reduced vestibular function can be detected. Persistent dizziness or lightheadedness or chronic dizziness has an important cortical representation in the frontal and parietal regions (Nigro et al., 2019). Noteworthy, research has found that $A \beta$ deposition and psychiatric outcomes can appear as early as 20 years before the first sign of AD, such as cognitive decline and memory loss (Dubois et al., 2016; Selkoe \& Hardy, 2016). Future, a study provided preliminary evidence that region-specific, mainly frontal and parietal regions, $A \beta$ deposition was present in some (but not all) depressive patients, especially in those with moderate-to-severe treatment resistance, and their depressive symptoms may represent prodromal manifestations of AD (Li et al., 2017).

Limitations of this study include the appropriate, valid outcome that detaches and measures the impact of dizziness or lightheadedness on health. It also includes a lack of uniform criteria for classification and definition of dizziness or lightheadedness in the medical records. However, we believe that 'unexplained' dizziness or lightheadedness may indicate functional dizziness, also known as psychogenic dizziness or persistent postural-perceptual dizziness (Kaski, 2020). Although the exact pathophysiology of functional dizziness is not yet clear, benign paroxysmal positional vertigo, vestibular migraine, and cerebral small vessel disease, anxiety disorders were the most contributing factors (Kaski, 2020). An acute 'dizzy episode' preceding these symptoms will be volunteered in many cases, and the disorder will usually emerge as this triggering event resolves. However, the processes are known to involve normal physiological and behavioral responses to an acute postural threat, which become inappropriately sustained after its remission. The usual response to dizziness or lightheadedness is to adopt protective balance strategies. Normal individuals demonstrate stiffening of posture and a shift in sensory information processing to increase reliance on visual and somatosensory cues in such situations. Those who develop a degree of functional dizziness or lightheadedness after an acute event show persistent high visual dependence, high anxiety, and hypervigilance to balance sensations compared with those 
who recover well. Prior anxiety and neurotic personality (state and trait anxiety) appear to pre-dispose to this maladaptation.

Remarkably, neuroimaging studies demonstrated that activity and connectivity in brain regions that process visual, vestibular, and spatial information are different between individuals with and without functional dizziness. The results suggest that a failure of cortical network (top-down) suppression of ascending postural information may result in persistence of the acute, high-risk postural behavior as well as high risk of anxiety and depressive symptomatology. Functional dizziness is, therefore, typically characterized by persistent dizziness and perceived instability or balance difficulty, worse in the upright position and in busy visual environments (Popkirov, Staab, \& Stone, 2018).

\section{Complexity and health of dizzy older adults who are at risk of $A D$}

The overall result of our study is, therefore, can be a valuable addition to current knowledge and may have clinical relevance, in that treatment response in dizzy patients with depression and/or anxiety could predict AD-related pathophysiology and aid clinicians in identifying patients in need of vigilant follow-up to assess cognitive functions. The suggested model for complexity and health of dizzy older adults who are at risk of $A D$ is presented in Fig. 2.

\section{Conclusions}

'Unexplained' dizziness or lightheadedness was associated with $A \beta$ plaque deposition among older adults. This association seems to be modulated by psychological outcomes. Although these symptoms can be linked by a common cause, it may suggest functional dizziness, also known as psychogenic dizziness or persistent postural-perceptual dizziness. Further studies are needed to support the findings.

\section{Declarations}

The author declares that there is no potential conflicts of interest or lack thereof

\section{References}

Alkadhi, K., \& Eriksen, J. (2011). The complex and multifactorial nature of Alzheimer's disease. Current neuropharmacology, 9(4), 586-586. doi:10.2174/157015911798376235

Best, C., Eckhardt-Henn, A., Tschan, R., \& Dieterich, M. (2009). Why do subjective vertigo and dizziness persist over one year after a vestibular vertigo syndrome? Ann N Y Acad Sci, 1164, 334-337. doi:10.1111/j.1749-6632.2009.03847.x

Bösner, S., Schwarm, S., Grevenrath, P., Schmidt, L., Hörner, K., Beidatsch, D., . . Haasenritter, J. (2018). Prevalence, aetiologies and prognosis of the symptom dizziness in primary care - a systematic review. 
Carmeli, E. (2015). Anxiety in the Elderly Can be a Vestibular Problem. Frontiers in public health, 3, 216216. doi:10.3389/fpubh.2015.00216

Chung, J. K., Plitman, E., Nakajima, S., Chakravarty, M. M., Caravaggio, F., Gerretsen, P., . . Graff-Guerrero, A. (2016). Cortical Amyloid $\beta$ Deposition and Current Depressive Symptoms in Alzheimer Disease and Mild Cognitive Impairment. J Geriatr Psychiatry Neurol, 29(3), 149-159. doi:10.1177/0891988715606230

Colledge, N., Lewis, S., Mead, G., Sellar, R., Wardlaw, J., \& Wilson, J. (2002). Magnetic resonance brain imaging in people with dizziness: a comparison with non-dizzy people. Journal of neurology, neurosurgery, and psychiatry, 72(5), 587-589. doi:10.1136/jnnp.72.5.587

Dubois, B., Hampel, H., Feldman, H. H., Scheltens, P., Aisen, P., Andrieu, S., ... Washington Dc, U. S. A. (2016). Preclinical Alzheimer's disease: Definition, natural history, and diagnostic criteria. Alzheimer's \& dementia : the journal of the Alzheimer's Association, 12(3), 292-323. doi:10.1016/j.jalz.2016.02.002

Evans, D. A., Funkenstein, H. H., Albert, M. S., Scherr, P. A., Cook, N. R., Chown, M. J., . . Taylor, J. O. (1989). Prevalence of Alzheimer's disease in a community population of older persons. Higher than previously reported. Jama, 262(18), 2551-2556.

Iwasaki, S., \& Yamasoba, T. (2014). Dizziness and Imbalance in the Elderly: Age-related Decline in the Vestibular System. Aging and disease, 6(1), 38-47. doi:10.14336/AD.2014.0128

Kamil, R. J., Bilgel, M., Wong, D. F., Resnick, S. M., \& Agrawal, Y. (2018). Vestibular Function and BetaAmyloid Deposition in the Baltimore Longitudinal Study of Aging. Frontiers in aging neuroscience, 10, 408-408. doi:10.3389/fnagi.2018.00408

Kaski, D. (2020). Neurological update: dizziness. Journal of neurology, 267(6), 1864-1869. doi:10.1007/s00415-020-09748-w

Kotova, O. V., \& Zamergrad, M. V. (2016). [Dizziness and anxiety disorders in the elderly]. Ter Arkh, 88(9), 131-134. doi:10.17116/terarkh2016889131-134

Li, P., Hsiao, I.-T., Liu, C.-Y., Chen, C.-H., Huang, S.-Y., Yen, T.-C., . . Lin, K.-J. (2017). Beta-amyloid deposition in patients with major depressive disorder with differing levels of treatment resistance: a pilot study. EJNMMI research, 7(1), 24-24. doi:10.1186/s13550-017-0273-4

Livingston, G., Huntley, J., Sommerlad, A., Ames, D., Ballard, C., Banerjee, S., . . Mukadam, N. (2020). Dementia prevention, intervention, and care: 2020 report of the Lancet Commission. Lancet, 396(10248), 413-446. doi:10.1016/s0140-6736(20)30367-6

Masters, C. L., \& Beyreuther, K. (1998). Alzheimer's disease. Bmj, 316(7129), 446-448. doi:10.1136/bmj.316.7129.446 
Mormino, E. C., \& Papp, K. V. (2018). Amyloid Accumulation and Cognitive Decline in Clinically Normal Older Individuals: Implications for Aging and Early Alzheimer's Disease. Journal of Alzheimer's disease : JAD, 64(s1), S633-S646. doi:10.3233/JAD-179928

Murphy, M. P., \& LeVine, H., 3rd. (2010). Alzheimer's disease and the amyloid-beta peptide. Journal of Alzheimer's disease : JAD, 19(1), 311-323. doi:10.3233/JAD-2010-1221

Neuhauser, H. K., Radtke, A., von Brevern, M., Lezius, F., Feldmann, M., \& Lempert, T. (2008). Burden of Dizziness and Vertigo in the Community. Archives of Internal Medicine, 168(19), 2118-2124.

doi:10.1001/archinte.168.19.2118

Nigro, S., Indovina, I., Riccelli, R., Chiarella, G., Petrolo, C., Lacquaniti, F., .. Passamonti, L. (2019). Reduced cortical folding in multi-modal vestibular regions in persistent postural perceptual dizziness. Brain Imaging and Behavior, 13(3), 798-809. doi:10.1007/s11682-018-9900-6

Previc, F. H. (2013). Vestibular loss as a contributor to Alzheimer's disease. Med Hypotheses, 80(4), 360367. doi:10.1016/j.mehy.2012.12.023

Qiu, C., Kivipelto, M., \& von Strauss, E. (2009). Epidemiology of Alzheimer's disease: occurrence, determinants, and strategies toward intervention. Dialogues in clinical neuroscience, 11(2), 111-128. doi:10.31887/DCNS.2009.11.2/cqiu

Roberts, R. O., Geda, Y. E., Knopman, D. S., Cha, R. H., Pankratz, V. S., Boeve, B. F., . . Rocca, W. A. (2008). The Mayo Clinic Study of Aging: design and sampling, participation, baseline measures and sample characteristics. Neuroepidemiology, 30(1), 58-69. doi:10.1159/000115751

Rodrigues, R., Smith, M. A., Wang, X., Perry, G., Lee, H. G., Zhu, X., \& Petersen, R. B. (2012). Molecular neuropathogenesis of Alzheimer's disease: an interaction model stressing the central role of oxidative stress. Future Neurol, 7(3), 287-305. doi:10.2217/fnl.12.27

Sachdev, P. S., Zhuang, L., Braidy, N., \& Wen, W. (2013). Is Alzheimer's a disease of the white matter? Curr Opin Psychiatry, 26(3), 244-251. doi:10.1097/YCo.0b013e32835ed6e8

Santos, C. Y., Snyder, P. J., Wu, W.-C., Zhang, M., Echeverria, A., \& Alber, J. (2017). Pathophysiologic relationship between Alzheimer's disease, cerebrovascular disease, and cardiovascular risk: A review and synthesis. Alzheimer's \& dementia (Amsterdam, Netherlands), 7, 69-87. doi:10.1016/j.dadm.2017.01.005

Selkoe, D. J., \& Hardy, J. (2016). The amyloid hypothesis of Alzheimer's disease at 25 years. EMBO molecular medicine, 8(6), 595-608. doi:10.15252/emmm.201606210

Sloane, P. D., Hartman, M., \& Mitchell, C. M. (1994). Psychological factors associated with chronic dizziness in patients aged 60 and older. J Am Geriatr Soc, 42(8), 847-852. doi:10.1111/j.1532-

5415.1994.tb06556.x 
Tinetti, M. E., Williams, C. S., \& Gill, T. M. (2000). Dizziness among older adults: a possible geriatric syndrome. Ann Intern Med, 132(5), 337-344. doi:10.7326/0003-4819-132-5-200003070-00002

Villemagne, V. L., Doré, V., Burnham, S., \& Rowe, C. C. (2021). A Imaging in Aging, Alzheimer's Disease, and Other Neurodegenerative Conditions. In R. A. J. O. Dierckx, A. Otte, E. F. J. de Vries, A. van Waarde, \& K. L. Leenders (Eds.), PET and SPECT in Neurology (pp. 283-343). Cham: Springer International Publishing.

Vlassenko, A. G., Benzinger, T. L. S., \& Morris, J. C. (2012). PET amyloid-beta imaging in preclinical Alzheimer's disease. Biochimica et biophysica acta, 1822(3), 370-379. doi:10.1016/j.bbadis.2011.11.005 Wiley, C. A., Lopresti, B. J., Venneti, S., Price, J., Klunk, W. E., DeKosky, S. T., \& Mathis, C. A. (2009). Carbon 11-labeled Pittsburgh Compound B and carbon 11-labeled (R)-PK11195 positron emission tomographic imaging in Alzheimer disease. Archives of neurology, 66(1), 60-67. doi:10.1001/archneurol.2008.511

\section{Figures}

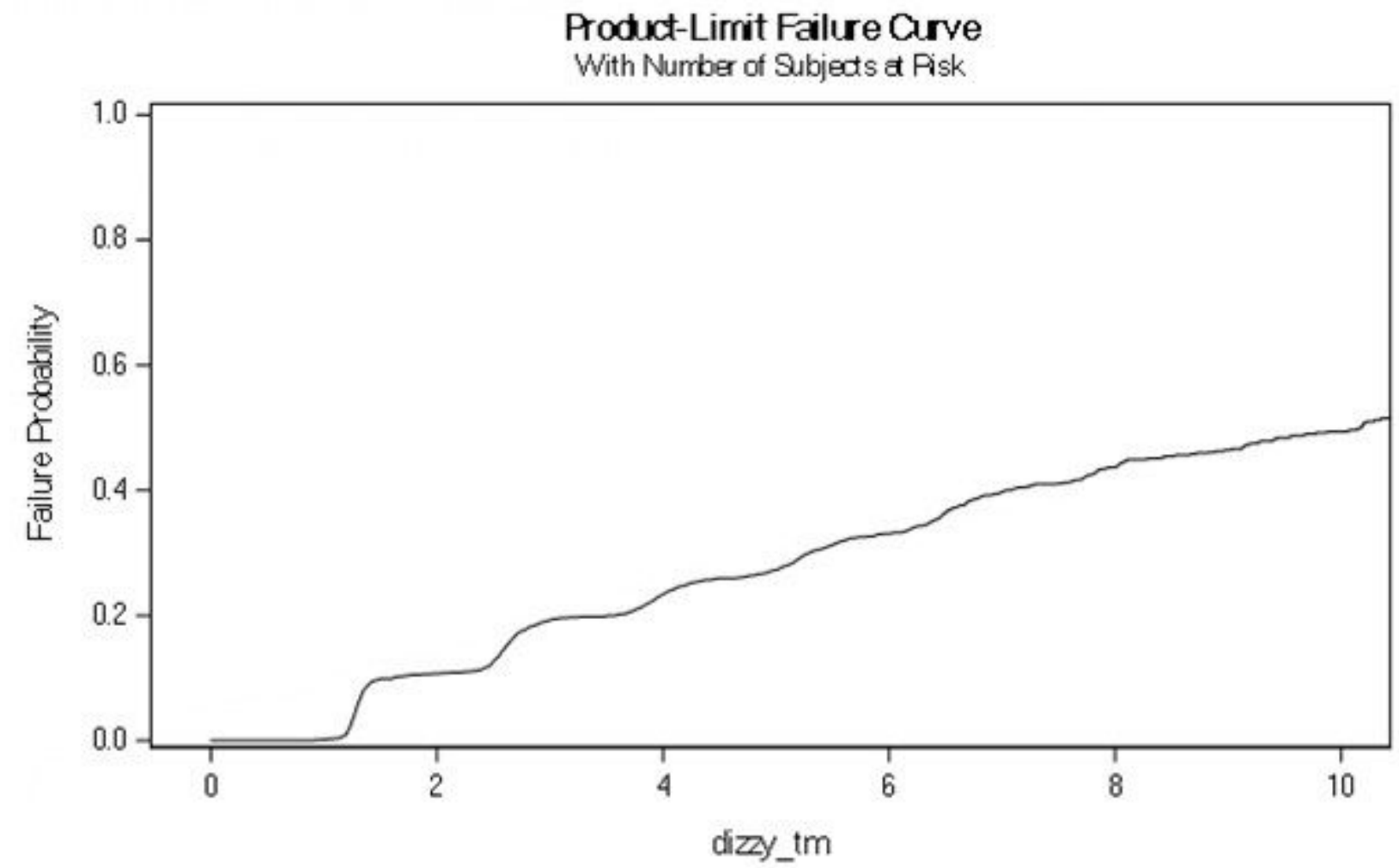

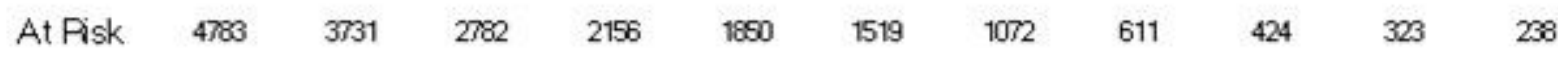

Figure 1 
Kaplan-Meier method: The 10-year risk of developing symptom of dizziness or lightheadedness in the group

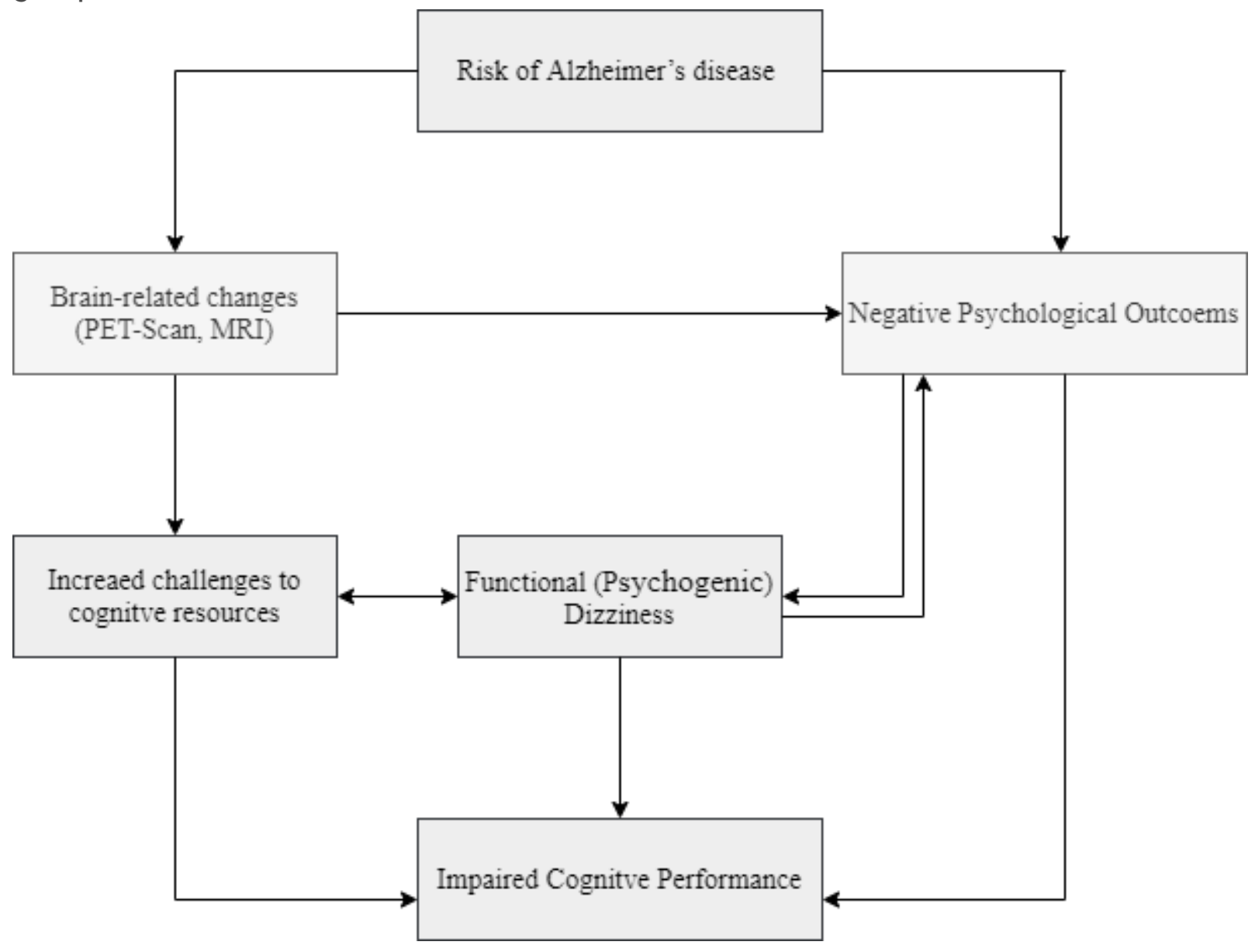

\section{Figure 2}

The author's suggested model Complexity and health of dizzy older adults who are at risk of AD 